\title{
Síndrome de Prader-Willi: Aspectos Metabólicos Associados ao Tratamento Com Hormônio de Crescimento
}

\section{artigo original}

\author{
JACK Y. KUO \\ VAÊ DITCHEKENIAN \\ THAIS D. MANNA \\ HILTON KUPERMAN \\ DURVAL DAMIANI \\ NuVARTE SetIAN
}

Unidade de Endocrinologia Pediátrica, Instituto da Criança, Hospital das Clínicas da

Faculdade de Medicina da Universidade de São Paulo USP, SP

Recebido em 04/07/05

Revisado em 22/02/06

Aceito em 19/05/06

\section{RESUMO}

Objetivos: Avaliar as alterações metabólicas de crianças e adolescentes portadores da Síndrome de Prader-Willi tratadas com hormônio de crescimento recombinante humano (rhGH). Casuística e métodos: Foram estudados sete pacientes: quatro meninos e três meninas, com idades de seis anos e seis meses a 14 anos e 11 meses. Receberam rhGH, 0,1 U/Kg/dia subcutâneo, seis vezes por semana, durante dois anos. Avaliamos dados antropométricos, lípides séricos, glicemia, IGF-I e composição corpórea, no início e após 12 e 24 meses de tratamento com rhGH. Resultados: Todos os pacientes tiveram elevação do IGF-I. Houve diminuição das pregas cutâneas, sendo que a média de perda de massa adiposa foi de $5,0 \%$ e a massa magra aumentou em média 7,6 kg nos prépúberes, e a paciente púbere ganhou $4,8 \mathrm{~kg}$ e teve um decréscimo de $5,6 \%$ de massa adiposa. Houve ganho de massa óssea de $0,07 \mathrm{~g} / \mathrm{cm}^{2}(7,3 \%)$ nos pré-púberes e de $0,02 \mathrm{~g} / \mathrm{cm}^{2}(2,0 \%)$ na menina púbere. Conclusão: Em nosso estudo, o uso do hormônio de crescimento na Síndrome de PraderWilli melhorou a composição corpórea e demonstrou aumento da massa muscular e massa óssea com melhora dos níveis lipídicos. (Arq Bras Endocrinol Metab 2007;51/1:92-98)

Descritores: Composição corpórea; Hormônio de crescimento; Obesidade; Síndrome de Prader-Willi

\section{ABSTRACT}

Prader-Willi Syndrome: Metabolic Aspects Related to Growth Hormone Treatment.

Aim: The focus of this study was to evaluate the metabolic profile of Prader-Willi Syndrome (PWS) patients treated with growth hormone. Patients and methods: Seven patients (four boys and three girls) with ages between six years and six months and 14 years and 11 months were treated with $\mathrm{GH} 0.1 \mathrm{U} / \mathrm{kg} /$ day subcutaneous by six times a week, for two years. Anthropometric data, lipids, glucose, IGF-I and body composition were evaluated at baseline and after 12 and 24 months. Results: IGF-I levels increased in all cases. Skin folds decreased. The mean reduction in body fat was $5.0 \%$ and the mean increased in lean mass was $7.6 \mathrm{~kg}$ in the prepubertal patients. The pubescent girl increased $4.8 \mathrm{~kg}$ and showed a $5.6 \%$ decrease in fat mass. A mean gain in the bone mass was $0.07 \mathrm{~g} / \mathrm{cm}^{2}(7.3 \%)$ in prepubescent cases, and $0.02 \mathrm{~g} / \mathrm{cm}^{2}(2.0 \%)$ in the pubescent girl. Conclusion: In our study GH treatment improved lean body and bone masses and had beneficial effect on lipid values. (Arq Bras Endocrinol Metab 2007;51/1:92-98)

Keywords: Body composition; Growth hormone; Obesity; Prader-Willi syndrome 
A ÍndRome de Prader-Willi (SPW) foi descrita em 1956 por Prader, Labhart e Willi (1). É uma doença genética devida à deleção da porção proximal do braço longo do cromossomo 15 paterno (15q11q13) ou, mais raramente, translocações, dissomia materna do cromossomo 15 ou anormalidades do imprinting cromossômico (2). A incidência é de cerca de 1:15.000 nascidos vivos e a prevalência de 60:1.000.000(3).

O quadro clínico se caracteriza por deficiência mental, hipotonia muscular, excesso de apetite, obesidade progressiva, baixa estatura, hipogonadismo, distúrbios do sono e do comportamento e características físicas inerentes à síndrome. Alguns destes sinais e sintomas podem estar relacionados com a secreção do hormônio de crescimento (GH) (3).

Após os quatro anos de idade, inicia-se a hiperfagia, a obesidade e os problemas de saúde (4). Na fase escolar nota-se dificuldade do aprendizado associada à deficiência mental, que pode ser leve ou moderada. Problemas comportamentais e sociais podem estar presentes, principalmente a compulsão alimentar (4), e a maioria dos pacientes é incapaz de ter uma vida independente (5). A obesidade é a complicação mais séria, tornando-se um fator de risco para o desenvolvimento de intolerância à glicose, doenças vasculares e respiratórias, reduzindo a expectativa de vida (5).

A redução da massa muscular presente na SPW agrava a hipotonia e contribui para o fraco desempenho físico (6).

O aspecto mais importante do tratamento é o controle do ganho excessivo de peso. Dietas hipocalóricas, medicamentos anoréticos e gastroplastias são ineficazes a longo prazo (7-10).

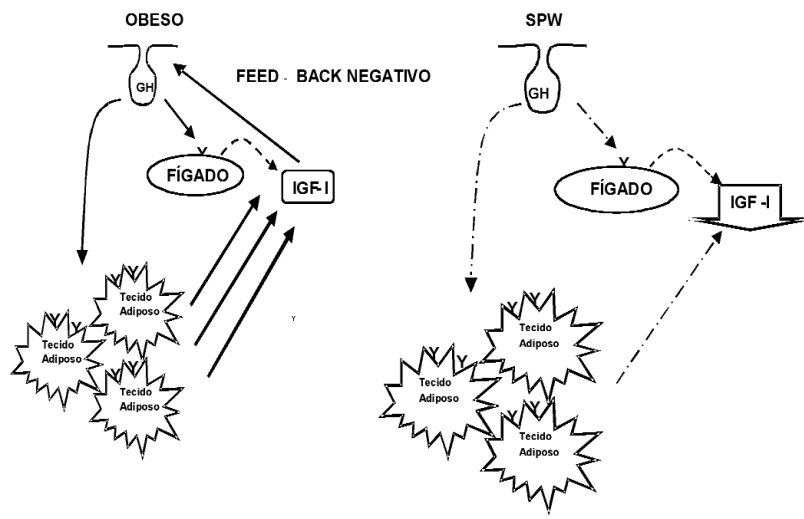

Figura 1. Irregularidade neurossecretória do hormônio de crescimento na Síndrome de Prader Willi.

$\mathrm{GH}$ : hormônio de crescimento; IGF-I: somatomedina [Modificado de Nam S.Y. (11)]
No obeso há um aumento da produção de fator de crescimento insulina símile (IGF-I), que é produzido pelo adipócito (11). Já na SPW, apesar de tecido adiposo abundante, não há um estímulo adequado de produção do IGF-I. A interpretação destes dados em função da secreção do GH é complicada pela obesidade do portador da SPW, uma vez que no obeso simples exógeno os níveis de $\mathrm{GH}$ circulantes estão menores, porém o IGF-I é normal e os níveis de IGFI livre são elevados. Na SPW, os níveis de IGF-I totais estão menores, o que indicaria uma situação de deficiência parcial de GH (11) (figura 1).

$\mathrm{O}$ acúmulo de triglicérides no tecido adiposo humano depende principalmente da lipase lipoprotéica (LLP), que promove a liberação de ácidos graxos livres (AGL) dos triglicérides transportados por lipoproteínas circulantes de muito baixo peso molecular (VLDL) e quilomícrons. A captação aumentada de AGL no tecido adiposo estimula o acúmulo de lipídios nos adipócitos (12). Em adipócitos incubados com hormônio de crescimento humano recombinante (rhGH) (13) e nos adipócitos humanos sob tratamento sistêmico com rhGH (14), há uma redução da atividade da LLP nas células do endotélio capilar adjacente ao tecido adiposo, diminuindo desta forma a oferta de AGL aos adipócitos, ao mesmo tempo que, no interior do adipócito, é ativada a lipase sensível aos hormônios favorecendo a hidrólise dos triglicérides e liberando ácidos graxos para a circulação (12) (figura 2). A capacidade de supressão da atividade da LLP pelo GH apresenta variação regional, podendo ser responsável pela redução mais pronunciada em depósitos gordurosos intra-abdominais quando comparada à gordura subcutânea (15) (figura 2).

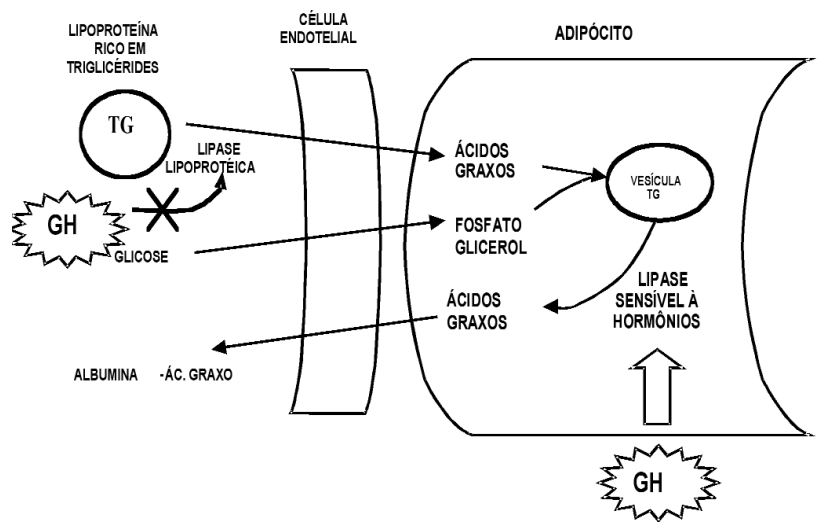

Figura 2. Ação do hormônio de crescimento no interior do adipócitos.

TG: triglicérides; GH: hormônio de crescimento [Modificado de Mahley, R.W. (12)] 
O comprometimento na altura final e a falta dos efeitos anabólico e lipolítico do hormônio de crescimento $(\mathrm{GH})$ estão relacionados à secreção reduzida do GH e conseqüentemente de IGF-I (16). O efeito lipolítico observado durante o tratamento com rhGH pode melhorar a composição corpórea com diminuição do tecido adiposo e aumento da massa muscular (16-21).

O objetivo deste trabalho foi analisar os efeitos do tratamento com rhGH na composição corpórea de crianças e adolescentes portadores de SPW, e assim contribuir para um conhecimento melhor dos aspectos metabólicos da síndrome, uma vez que os resultados mostrados na literatura têm aspectos nem sempre concordantes.

\section{CASUÍSTICA e MÉTODOS}

\section{Casuística}

Foram avaliados sete pacientes durante dois anos (quatro do sexo masculino e três do feminino) com idades de 6 anos e 6 meses a 14 anos e 11 meses. Critério de inclusão: confirmação genética do diagnóstico clínico. Critérios de exclusão: portadores de doenças sistêmicas, uso de anorexígenos, diabetes mellitus, problemas ósseos, musculares e deficiência neurológica graves.

Iniciamos o trabalho com sete pacientes, porém dois deles foram excluídos por apresentarem efeitos colaterais ao tratamento (epifisiólise em um e edema e hipertensão arterial em outro).

\section{Métodos}

Os pacientes foram avaliados a cada três meses, no ambulatório de Endocrinologia Pediátrica do Instituto da Criança da Faculdade de Medicina da Universidade de São Paulo. Determinou-se o peso (P) em balança digital (Dixtal); altura (A) em estadiômetro de madeira, espessuras ( $\mathrm{mm}$ ) das pregas cutâneas (PC) tricipital, dorsal e abdominal, com plicômetro de metal de acordo com Frisancho (22); índice de massa corpórea (IMC) (23); velocidade de crescimento; $\mathrm{z}$-escore de peso e estatura. As curvas de referência utilizadas foram as do NCHS e os cálculos, efetuados através do programa EPI INFO 2000 (24). Foram avaliados antes e aos 12 e 24 meses após o tratamento com rhGH a composição corpórea pela técnica DEXA (dual energy $X$-ray absorptiometry), com determinação da massa adiposa, massa magra e densidade mineral óssea.

As glicemias foram obtidas pelo método da hexoquinase, hemoglobina glicada por cromatografia de troca iônica, IGF-I por radioimunoensaio e perfil lipídico (colesterol total e frações e triglicérides) por colorimetria. O GH foi dosado por método imunofluorimétrico, e os testes de estímulo para $\mathrm{GH}$ foram realizados com clonidina $(0,15$ $\mathrm{mg} / \mathrm{m}^{2}$ v.o. $)$ e insulina $(0,1 \mathrm{U} / \mathrm{kg}$ via endovenosa $)$; valores de pico acima de 5,0 $\mathrm{ng} / \mathrm{ml}$ foram considerados normais
(25). A idade óssea foi avaliada pelos critérios de Greulich e Pyle (26).

O rhGH na dose $0,1 \mathrm{U} / \mathrm{kg} /$ dia subcutâneo, no máximo $8 \mathrm{U} /$ dia, foi administrado seis vezes por semana por 2 anos, com consentimento escrito e informado dos pais e aprovado pelo Comitê de Ética da Faculdade de Medicina da Universidade de São Paulo e do Instituto da Criança.

A análise dos resultados é descritiva e os valores obtidos são apresentados por médias, valores máximos e mínimos, percentuais, do grupo de quatro pacientes pré-púberes e uma púbere.

\section{RESULTADOS}

A média da velocidade de crescimento antes do tratamento nos pacientes pré-púberes foi de $5,1 \mathrm{~cm} / \mathrm{ano}$, de $9,4 \mathrm{~cm}$ após 12 meses e $5,7 \mathrm{~cm}$ no segundo ano de tratamento, respectivamente. A paciente púbere apresentou um aumento de $5,7 \mathrm{~cm}$ no primeiro ano. Após dois anos de tratamento, a média de acréscimo do zescore da estatura para a idade cronológica nos pacientes pré-púberes foi de 0,7 e da paciente púbere, de 0,6 (tabela 1 ).

Após dois anos de rhGH, a queda do z-escore para peso para a idade nos pacientes pré-púberes e púbere foi de 0,8 e 0,1 , respectivamente, e z-escore do IMC foi de 0,39 e de 0,23 (tabela 1). Nos pré-púberes, a soma das pregas cutâneas (tricipital, dorsal e abdominal) apresentou uma diminuição de 41,9 mm após dois anos de tratamento com rhGH, enquanto na paciente púbere a diminuição foi de $30,5 \mathrm{~mm}$ (tabela 1 ).

A média de massa adiposa total dos quatros pacientes pré-púberes antes do rhGH foi de $51,9 \%$, reduzindo-se para 46,9\% (5,0\%) após dois anos de tratamento. O paciente 1 apresentou a maior perda observada nos pré-púberes: de $56,5 \%$ para $45,5 \%$ (11\%), e a paciente púbere teve sua massa adiposa reduzida de $63,0 \%$ para $47,4 \%(15,6 \%)$ (tabela 1 ).

$\mathrm{O}$ ganho médio de massa magra dos pacientes pré-púberes foi de 7,6 $\mathrm{kg}$ e da paciente púbere, de 4,8 $\mathrm{kg}$ (tabela 1).

A massa óssea apresentou um aumento médio de $0,07 \mathrm{~g} / \mathrm{cm}^{2}(7,3 \%)$ nos pré-púberes e na púbere, de $0,002 \mathrm{~g} / \mathrm{cm}^{2}(2,0 \%)$ (tabela $\left.\mathrm{l}\right)$.

Após dois anos de tratamento nos pacientes pré-púberes, houve uma queda de $20,6 \%$ do colesterol total e de $34,9 \%$ no colesterol LDL, e um aumento de $34,9 \%$ no colesterol HDL. A queda dos triglicérides (TG) foi de $43,1 \%$, e na paciente púbere houve aumento de $26,9 \%$ no colesterol HDL (tabela 2 ).

$\mathrm{O}$ z-escore para IGF-I no início do tratamento foi de - 0,8 . Após um ano de uso do rhGH, foi para 1,4 
Tabela 1. Características dos dados físicos e antropométricos após 2 anos de tratamento com hormônio de crescimento recombinante.

\begin{tabular}{|c|c|c|c|c|c|c|c|c|c|c|c|c|}
\hline Pacientes & $\begin{array}{l}\text { Tempo de } \\
\text { tratamento }\end{array}$ & IC & 10 & Estatura & $\begin{array}{l}\text { Veloci- } \\
\text { dade de } \\
\text { Cresci- } \\
\text { mento }\end{array}$ & $\begin{array}{l}\text { Estatura/ } \\
\text { Idade }\end{array}$ & $\begin{array}{l}\text { Peso/ } \\
\text { Idade }\end{array}$ & IMC & $\begin{array}{c}\text { Pregas } \\
\text { Cutâneas } \\
\text { Total }\end{array}$ & $\begin{array}{c}\text { Massa } \\
\text { adiposa } \\
\text { Total }\end{array}$ & $\begin{array}{c}\text { Massa } \\
\text { magra } \\
\text { Total }\end{array}$ & $\begin{array}{l}\text { Massa } \\
\text { óssea }\end{array}$ \\
\hline sexo & 2 anos & & & $\mathbf{c m}$ & cm/ano & Z-escore & Z-escore & Z-escore & $\mathbf{m m}$ & em \% & $\mathbf{k g}$ & $\begin{array}{c}\mathrm{g} / \mathrm{cm}^{2} \\
(\%)\end{array}$ \\
\hline 1 & início & $6 a 6 m$ & $7 a$ & 119,4 & 6,9 & 0,2 & 6,7 & 3,13 & 114,0 & 56,5 & 17,0 & 0,888 \\
\hline M & $1^{\circ}$ ano & & $10 \mathrm{a} 6 \mathrm{~m}$ & 131,0 & 11,6 & 1,4 & 5,2 & 2,59 & 76,5 & 49,9 & 24,0 & 0,986 \\
\hline \multirow[t]{2}{*}{ Pré-púbere } & $2^{\circ}$ ano & & $11 a$ & 139,2 & 8,2 & 1,9 & 5,0 & 2,33 & 57,5 & 45,5 & 26,6 & 0,966 \\
\hline & Incremento final & & $4 a$ & 19,8 & & 1,7 & $-1,7$ & $-0,80$ & $-56,5$ & $-11,0$ & 9,6 & $0,078(8,8 \%)$ \\
\hline 2 & início & $7 a$ & $7 a 6 m$ & 113,6 & 3,6 & $-1,5$ & 1,0 & 1,95 & 83,0 & 37,0 & 15,7 & 0,809 \\
\hline M & $1^{\circ}$ ano & & $10 \mathrm{a} 6 \mathrm{~m}$ & 120,0 & 6,4 & $-1,2$ & 0,8 & 1,66 & 57,0 & 37,7 & 17,6 & 0,824 \\
\hline \multirow[t]{2}{*}{ Pré-púbere } & $2^{\circ}$ ano & & $11 a$ & 124,2 & 4,2 & $-1,3$ & 0,4 & 1,35 & 53,0 & 31,5 & 20,3 & 0,811 \\
\hline & Incremento final & & $3 a 6 m$ & 10,6 & & 0,2 & $-0,6$ & $-0,60$ & $-30,0$ & $-5,5$ & 4,6 & $0,002(0,2 \%)$ \\
\hline 3 & início & 10a $1 \mathrm{~m}$ & $13 a$ & 137,3 & 4,7 & $-0,02$ & 5,65 & 2,72 & 108,0 & 59,1 & 28,2 & 1,111 \\
\hline \multirow[t]{2}{*}{ Pré-púbere } & $2^{\circ}$ ano & & $14 a$ & 152,2 & 5,2 & 0,32 & 4,45 & 2,57 & 76,0 & 55,2 & 37,5 & 1,178 \\
\hline & Incremento final & & $1 \mathrm{a}$ & 14,9 & & 0,3 & $-1,1$ & $-0,15$ & $-32,0$ & $-3,9$ & 9,3 & $0,067(6,0 \%)$ \\
\hline 4 & início & 11a $9 m$ & $13 a$ & 132,6 & 5,1 & $-2,1$ & 2,1 & 2,45 & 138,0 & 54,9 & 25,4 & 1,021 \\
\hline M & $1^{\circ}$ ano & & $13 a$ & 142,5 & 9,9 & $-1,5$ & 2,2 & 2,41 & 111,0 & 62,0 & 25,3 & 1,148 \\
\hline \multirow[t]{2}{*}{ Pré-púbere } & $2^{\circ}$ ano & & $14 a$ & 147,7 & 5,2 & $-1,6$ & 2,3 & 2,45 & 89,0 & 55,2 & 32,3 & 1,166 \\
\hline & Incremento final & & $1 \mathrm{a}$ & 15,1 & & 0,5 & 0,2 & 0,00 & $-49,0$ & 0,3 & 6,8 & $0,145(14,2 \%)$ \\
\hline início & $8 a 10 m$ & $10 \mathrm{a} 1 \mathrm{~m}$ & 125,7 & 5,1 & $-0,9$ & 3,8 & 2,56 & 110,8 & 51,9 & 21,6 & 0,96 & \\
\hline Média dos 4 & $1^{\circ}$ ano & & 11a $9 \mathrm{~m}$ & 135,1 & 9,4 & $-0,3$ & 3,1 & 2,29 & 80,1 & 51,8 & 24,3 & 1,03 \\
\hline \multirow[t]{2}{*}{ Pré-púberes } & $2^{\circ}$ ano & & $12 \mathrm{a} 9 \mathrm{~m}$ & 140,8 & 5,7 & $-0,2$ & 3,0 & 2,18 & 68,9 & 46,9 & 29,2 & 1,03 \\
\hline & Incremento final & & 2a $7 \mathrm{~m}$ & 15,1 & & 0,7 & $-0,8$ & $-0,39$ & $-41,9$ & $-5,0$ & 7,6 & $0,07(7,3 \%)$ \\
\hline 5 & início & 14a $1 \mathrm{~m}$ & $14 a 6 m$ & 145,8 & 3 & $-2,4$ & 1,7 & 2,22 & 81,0 & 63 & 27,4 & 1,085 \\
\hline púbere & $1^{\circ}$ ano & & $14 a 6 m$ & 150,0 & 4,2 & $-1,8$ & 1,3 & 1,93 & 47,2 & 54,2 & 31,2 & 1,084 \\
\hline \multirow[t]{2}{*}{$\mathrm{F}$} & $2^{\circ}$ ano & & $15 a$ & 151,5 & 1,5 & $-1,8$ & 1,6 & 1,99 & 50,5 & 57,4 & 32,1 & 1,107 \\
\hline & Incremento final & & $6 m$ & 5,7 & & 0,6 & $-0,1$ & $-0,23$ & $-30,5$ & $-5,6$ & 4,8 & $0,022(2,0 \%)$ \\
\hline
\end{tabular}

M: masculino; F: feminino; a: anos; m: meses; IC: idade cronológica; IO: idade óssea; IMC: índice de massa corpórea. Incremento final= (valor inicial - valor no $2^{\circ}$ ano).

Tabela 2. Evolução do perfil lipídico, IGF-I e hemoglobina glicada após 2 anos de tratamento com hormônio de crescimento.

\begin{tabular}{|c|c|c|c|c|c|c|c|c|}
\hline PACIENTE & & $\begin{array}{l}\text { Colesterol } \\
\text { mg/dL }\end{array}$ & $\begin{array}{c}\text { HDL } \\
\mathrm{mg} / \mathrm{dL}\end{array}$ & $\begin{array}{c}\text { LDL } \\
\mathrm{mg} / \mathrm{dL}\end{array}$ & $\begin{array}{l}\text { VLDL } \\
\mathrm{mg} / \mathrm{dL}\end{array}$ & $\begin{array}{c}\text { Triglicérides } \\
\text { mg/dL }\end{array}$ & $\begin{array}{c}\text { IGF-I } \\
\text { z-escore }\end{array}$ & $\begin{array}{c}\text { Hb A1c } \\
\text { mg/dL }\end{array}$ \\
\hline \multirow[t]{4}{*}{1} & início & 192,0 & 50,0 & 117,0 & 25,0 & 124,0 & 0,2 & 5,1 \\
\hline & $1^{\circ}$ ano & 173,0 & 55,0 & 105,0 & 13,0 & 67,0 & 3,5 & 5,6 \\
\hline & $2^{\circ}$ ano & 171,0 & 59,0 & 101,0 & 11,0 & 54,0 & 4,8 & 5,0 \\
\hline & Incremento final & $-21,0$ & 9,0 & $-16,0$ & $-14,0$ & $-70,0$ & 4,6 & \\
\hline$\%$ & & $-10,9 \%$ & $+18,0 \%$ & $-13,7 \%$ & $-56,0 \%$ & $-56,5 \%$ & & \\
\hline \multirow[t]{4}{*}{2} & início & 183,0 & 35,0 & 115,0 & 33,0 & 163,0 & $-0,6$ & 5,6 \\
\hline & $1^{\circ}$ ano & 116,0 & 33,0 & 70,0 & 13,0 & 64,0 & 0,3 & 6,2 \\
\hline & $2^{\circ}$ ano & 132,0 & 53,0 & 68,0 & 11,0 & 55,0 & 1,7 & 6,0 \\
\hline & Incremento final & $-51,0$ & 18,0 & $-47,0$ & $-22,0$ & $-108,0$ & 2,3 & \\
\hline$\%$ & & $-27,9 \%$ & $+51,4 \%$ & $-40,9 \%$ & $-66,7 \%$ & $-66,3 \%$ & & \\
\hline \multirow[t]{4}{*}{3} & início & 222,0 & 40,0 & 168,0 & 14,0 & 71,0 & $-1,7$ & 6,1 \\
\hline & $1^{\circ}$ ano & 179,0 & 42,0 & 116,0 & 21,0 & 105,0 & 0,6 & 5,4 \\
\hline & $2^{\circ}$ ano & 183,0 & 47,0 & 122,0 & 14,0 & 68,0 & 2,1 & 5,7 \\
\hline & Incremento final & $-39,0$ & 7,0 & $-46,0$ & 0,0 & $-3,0$ & 3,8 & \\
\hline$\%$ & & $-17,6 \%$ & $+7,5 \%$ & $-27,4 \%$ & $0,0 \%$ & $-4,2 \%$ & & \\
\hline \multirow[t]{4}{*}{4} & início & 174,0 & 40,0 & 120,0 & 14,0 & 68,0 & $-1,1$ & 5,7 \\
\hline & $1^{\circ}$ ano & 143,0 & 77,0 & 52,8 & 13,2 & 66,0 & 1,1 & 5,6 \\
\hline & $2^{\circ}$ ano & 129,0 & 61,0 & 60,6 & 7,4 & 37,0 & 1,2 & 4,9 \\
\hline & Incremento final & $-45,0$ & 21,0 & $-59,4$ & $-6,6$ & $-31,0$ & 2,3 & \\
\hline$\%$ & & $-25,9 \%$ & $+52,5 \%$ & $-49,5 \%$ & $-47,1 \%$ & $-45,6 \%$ & & \\
\hline
\end{tabular}

Incremento final $=\left(\right.$ valor do $2^{\circ}$ ano - valor do início $)$

Valores de referência: Colesterol Total < $170 \mathrm{mg} / \mathrm{dL} ; \mathrm{LDL}<110 \mathrm{mg} / \mathrm{dL} ; \mathrm{HDL}>39 \mathrm{mg} / \mathrm{dL} ;$ Triglicérides $<101 \mathrm{mg} / \mathrm{dL}$ de acordo com NCEP (National Cholesterol Education Program) (45). 
Tabela 3. Valores do GH após teste de estímulo.

\begin{tabular}{|c|c|c|c|c|c|c|c|c|}
\hline & TESTE/PACIENTE & 1 & 2 & 3 & 4 & média & d.p. & 5* \\
\hline Pico máximo de $\mathrm{GH}$ & Teste de Clonidina & 0,2 & 0,2 & 1,8 & 1,7 & 0,8 & 0,8 & 0,1 \\
\hline em $(n g / m L)$ & Teste de Insulina & 6,6 & 3,8 & 0,7 & 0,3 & 2,9 & 2,6 & 9,7 \\
\hline
\end{tabular}

Valor de referência para o método imunofluorimétrico maior que $5 \mathrm{ng} / \mathrm{ml}$ (25).

* púbere

e após dois anos, para 2,5. A média de incremento em dois anos foi de 3,3 (tabela 2 ).

Os níveis do pico de GH nos testes de estímulo com clonidina e insulina estavam baixos em todos os pacientes (tabela 3 ).

A média de avanço de idade óssea no período de dois anos foi de dois anos e sete meses (tabela 1 ).

\section{DISCUSSÃo}

Na SPW, a obesidade mórbida e a hipotonia muscular contribuem para redução da sobrevida, limitada geralmente pelas duas primeiras décadas de vida (5). A compreensão das alterações metabólicas destas crianças e adolescentes pode fornecer sugestões importantes para o tratamento.

Nos pacientes deste estudo, a obesidade se mostrou presente em todos, assim como a compulsão pelos alimentos.

Nos testes de estímulo de GH com clonidina e insulina, nenhum paciente apresentou pico de $\mathrm{GH}$ acima de $10 \mathrm{ng} / \mathrm{ml}$; um dos pacientes pré-púbere apresentou $6,6 \mathrm{ng} / \mathrm{ml}$ e a púbere, $9,7 \mathrm{ng} / \mathrm{ml}$. Considerando o valor de corte de $5 \mathrm{ng} / \mathrm{ml}$, três pacientes foram não responsivos. Vários autores descrevem a secreção diminuída de GH na SPW como uma irregularidade neurossecretória $(16,27)$.

$\mathrm{O}$ tratamento destes pacientes com rhGH pode melhorar a estatura final, porém a grande contribuição do GH tem sido a melhora da composição corpórea, minimizando as conseqüências clínicas desta síndrome (28-30). Em nossos pacientes, observamos aumento da velocidade de crescimento, principalmente no primeiro ano de tratamento, porém acompanhado de um avanço proporcional na idade óssea.

$\mathrm{O}$ tratamento com rhGH não proporcionou perda de peso em termos absolutos, porém levou a um ganho menor neste período, com discreta diminuição do índice de massa corpórea. Todas as pregas cutâneas tiveram uma diminuição após dois anos de tratamento.

A composição corpórea das crianças com a SPW se assemelha aos estados de deficiência de $\mathrm{GH}$, por apresentarem massa magra reduzida e massa adiposa aumentada $(31,32)$, em contraste com os obesos, que apresentam aumento da massa magra e adiposa (33). Três estudos $(31,34,35)$ envolvendo indivíduos jovens com a SPW, mostraram massa adiposa excessiva de $42 \%(n=14), 51 \%(n=5)$ e $47 \%(n=27)$. Nossos resultados mostraram a média de massa adiposa nas crianças pré-púberes de $51,9 \%$ e na púbere, de $63 \%$, portanto semelhantes aos dados da literatura. Em crianças normais, a massa adiposa é aproximadamente $15 \%$ do peso corpóreo em ambos sexos até os sete anos, e cerca de $18 \%$ aos 10 anos (36). Em adultos normais do sexo masculino, a porcentagem de gordura corpórea é de $18 \%$ e no sexo feminino, $22 \%$ (37).

Encontramos valores de massa adiposa extremamente elevados, acentuados pela falta do GH e por outros fatores tais como a compulsão alimentar e distúrbios hipotalâmicos, ainda não totalmente caracterizados $(38,39)$. Observamos que o tratamento com rhGH proporcionou uma discreta melhora na composição corpórea, porém o tratamento assume uma importância maior por bloquear o ganho excessivo da gordura corpórea, pois todos tiveram aumento da massa magra e do tecido ósseo.

A melhora do desempenho motor e da agilidade também foi documentada em crianças com a SPW que receberam rhGH (18). Os resultados sugerem que o tratamento propicia efeitos benéficos na aparência física, energia e resistência, melhorando assim a inserção psicossocial das crianças afetadas $(16,29,40)$. Muitas destas observações foram relatadas espontaneamente pelos pais. Observamos que a melhora começa a ser citada pelos pais após os primeiros seis meses de tratamento, principalmente em seu rendimento físico e comportamento psicossocial, com atividades anteriormente não imagináveis, tais como o uso da bicicleta e atividades cotidianas normais. Outros trabalhos citam bons resultados do tratamento com rhGH na função respiratória, com aumento da ventilação e da sensibilidade dos quimio-receptores periféricos ao dióxido de carbono, sugerindo que o rhGH exerça um efeito no sistema regulador respiratório central (4l). Dados sobre os efeitos a longo prazo do rhGH na composição corporal são, entretanto, limitados, e os resultados recentes mostram que quatro a cinco anos de trata- 
mento com rhGH podem ainda ser insuficientes para normalizar e estabilizar a massa magra e o IMC $(19,20)$.

Em relação ao perfil lipídico, houve uma normalização dos triglicérides, que estavam elevados em dois pacientes, e do colesterol total às custas da queda do LDL e aumento do HDL.

O tratamento com rhGH promove a queda dos níveis do colesterol total às custas do colesterol de baixa densidade (LDL) e de muito baixa densidade (VLDL), pela atuação do hormônio diretamente no receptor hepático das apolipoproteínas B e promovendo a metabolização e remoção destas lipoproteínas (42). Esta melhora no perfil lipídico pode contribuir para a redução do risco de doenças cardiovasculares associadas à obesidade.

Em relação ao metabolismo de hidratos de carbono, não observamos alterações da glicemia e da hemoglobina glicada (tabela 2). Estudos mostram que a glicemia tende a permanecer dentro da faixa de normalidade ou alterada durante o tratamento com rhGH, principalmente nos pacientes previamente obesos que recebem altas doses, havendo necessidade de monitorização constante da glicemia. Os níveis de insulina em crianças com SPW são menores do que em controles obesos, mas aumentam durante o tratamento $(43)$.

Os efeitos adversos, relatados durante o tratamento com rhGH em pacientes com SPW, são geralmente similares aos observados nas crianças com deficiência de hormônio de crescimento clássica, síndrome de Turner ou insuficiência renal crônica. Foram observados em três pacientes efeitos colaterais, edema nos membros inferiores em um, hipertensão arterial e edema nos membros inferiores em outro, e epifisiólise da cabeça do fêmur em outro. Um deles apresentou regressão do edema com a suspensão do rhGH e reintrodução gradativa. O paciente que apresentou epifisiólise, fato não descrito na literatura em pacientes portadores da SPW e o outro com edema e hipertensão arterial persistente, foram excluídos.

Portanto, concluímos que os pacientes portadores da SPW podem apresentar uma possível irregularidade neurossecretória do $\mathrm{GH}$ e o tratamento com rhGH melhorou a composição corpórea, diminuiu a massa adiposa e aumentou a massa magra e óssea. Estabilizou-se o índice de massa corpórea, houve aumento da velocidade de crescimento, principalmente no primeiro ano de tratamento, com correspondente avanço da maturação óssea e elevação dos níveis de IGF-I. A literatura também confirma que o uso de rhGH é capaz de normalizar o IGF-I (44). Os níveis dos triglicérides normalizaram-se e houve uma queda do colesterol LDL e aumento do HDL. Não observamos alterações nos níveis glicêmicos e da hemoglobina glicada.

Os aspectos mais importantes do tratamento dos pacientes com SPW são o controle do ganho excessivo de peso e a melhora da composição corpórea, que colabora para diminuição da morbidade e mortalidade desses pacientes. Segundo relato dos familiares, os pacientes tiveram nítida melhora no desempenho motor, mais agilidade, atenuando as dificuldades sociais.

\section{AGRADECIMENTOS}

Ao Dr. Eduardo Meirelles, responsável pela Unidade de Densitometria Óssea do Instituto de Ortopedia da Faculdade de Medicina da Universidade de São Paulo. E ao Dr. Ulysses Dória, pela orientação estatística.

\section{REFERÊNCIAS}

1. Prader A, Labhart A, Willi H. Ein Syndrom von Adipositas, Kleinwuchs, Kryptorchismus und Oligophrenie nach myotonieartigem Zustand im Neugeborenenalter. Schweiz Med Wochenschr 1956;86:1260-1.

2. Gillessen-Kaesbach G, Gross S, Kaya-Westerloh S, Passarge $E$, Horsthemke B. DNA methylation based testing of 450 patients suspected of having Prader-Willi syndrome. J Med Genet 1995;32:88-92.

3. Holm V, Cassidy SB, Butler MG, Hanchett JM, Greenswag LR, Whitmann BYGF. Prader-Willi syndrome: consensus diagnostic criteria. Pediatrics 1993;91:398-402.

4. Cassidy SB. Prader-Willi syndrome. J Med Genet 1997;34:917-23.

5. Greenswag L. Adults with Prader-Willi syndrome. A survey of 232 cases. Dev Med Child Neurol 1987;29:145-52.

6. Brambilla P, Bosio L, Manzoni P, Pietrobelli A, Beccaria L, Chiumello G. Peculiar body composition in patients with PraderWilli syndrome. Am J Clin Nutr 1997;65:1369-74.

7. Nelson RA, Huse DM, Holman RT. Nutrition, metabolism, body composition and response to the Ketogenic diet in Prader-Willi Syndrome. In: Holm VA, Sulzbacher S, Pipes PL (eds.). Prader-Willi Syndrome, chapt 8. University Baltimore: Park Press, 1981. pp. 105-20.

8. Selikowitz M, Summan J, Pendergast A, Wright S. Fenfluramine in Prader-Willi Syndrome: a double-blind, placebocontrolled trial. Arch Dis Child 1990;65:112-4.

9. Marinari G, Gianella E, Adami G, Gandllo P, Scopinoro N. Long-term results of bileopancreatic diversion in subjects with Prader-Willi Syndrome Obes Surg 1996;6:303-4.

10. Soper RT, Mason EE, Printen KJ, Zellweger H. Surgical treatment of morbid obesity in Prader-Willi Syndrome. In: Holm VA, Sulzbacher S, Pipes PL (eds). Prader-Willi Syndrome. Baltimore: University Park Press, 1981. pp. 121-31.

11. Nam SY, Marcus C. Growth hormone and adipocyte function in obesity. Horm Res 2000;53(suppl):87-97.

12. Mahley RW, Weisgraber KH, Farese RV. Disorders of lipid metabolism. In: Wilson JD Foster, DW, Kronenberg HM, Larese PR. Williams textbook of endocrinology. $9^{\text {th }}$ ed. Philadelphia: Saunders, 1998, pp. 1102-3. 
13. Dietz J, Schwartz J. Growth hormone alters lipolysis and hormone-sensitive lipase activity in 3T3-F442A adipocytes. Metabolism 1991;40(8):800-6.

14. Ottosson M, Vikman-Adolfsson K, Enerback S, Elander A, Bjorntorp P, Eden S. Growth hormone inhibits lipoprotein lipase activity in human adipose tissue. J Clin Endocrinol Metab 1995;80(3):934-41.

15. Johannsson G, Marin P, Lonn L, Ottosson M, Stenlof K, Bjorntorp $\mathrm{P}$, et al. Growth hormone treatment of abdominally obese men reduces abdominal fat mass, improves glucose and lipoprotein metabolism, and reduces diastolic blood pressure. J Clin Endocrinol Metab 1997;82(3):727-34

16. Burman $P$, Ritzen EM, Lindgren AC. Endocrine dysfunction in Prader-Willi syndrome: a review with special reference to $\mathrm{GH}$. Endocr Rev 2001;22(6):787-99.

17. Hauffa BP. One-year results of growth hormone treatment of short stature in Prader-Willi syndrome. Acta Paediatr Suppl 1997;423:63-5.

18. Myers SE, Carrel AL, Whitman BY, Allen DB. Sustained benefit after 2 years of growth hormone on body composition, fat utilization, physical strength and agility, and growth in Prader-Willi syndrome. J Pediatr 2000;137:42-9.

19. Carrel AL, Myers SE, Whitman BY, Allen DB. Benefits of longterm GH therapy in Prader-Willi syndrome: a 4-year study. J Clin Endocrinol Metab 2002;87(4):1581-5.

20. Obata K, Sakazume S, Yoshino A, Murakami N, Sakuta R. Effects of 5 years growth hormone treatment in patients with Prader-Willi syndrome. J Pediatr Endocrinol Metab 2003;16(2):155-62.

21. Hoybye C. Endocrine and metabolic aspects of adult PraderWilli syndrome with special emphasis on the effect of growth hormone treatment. Growth Horm IGF Res 2004;14(1):115.

22. Frisancho A. Anthropometric standards for the assessment of growth and nutritional status. Ann Arbor: University of Michigan Press, 1993. p. 32.

23. Rolland-Cachera MF. Body composition during adolescence: methods, limitations and determinations. Horm Res 1993;39:25.

24. EPI INFO 2000 Division of Nutrition. Center for Chronic Disease Prevention and Health Promotion. Centers for Disease Control and Prevention 1600 Clifton Road NE, MS A08. Atlanta, GA 30333 U.S.A. Nutrition Unit. World Health Organization. 1211 Geneva 27. Switzerland (www. CDC.com)

25. Reiter EO, Rosenfeld RG. Normal and aberrant growth. In: Wilson JD, Foster DW, Kronenberg HM, Larese PR. Williams textbook of endocrinology. $9^{\text {th }}$ ed. Philadelphia: Saunders, 1998. pp. 1471-3.

26. Greulich WW, Pyle ST. Radiological Skeletal Development of the Hand and Wrist. Stanford: Stanford University Press, 1959.

27. Corrias A, Bellone J, Beccaria L, Bosio L, Trifiro G, Livieri C, et al. GH/IGF-I axis in Prader-Willi syndrome: evaluation of IGFI levels and of the somatotroph responsiveness to various provocative stimuli. Genetic Obesity Study Group of Italian Society of Pediatric Endocrinology and Diabetology. J Endocrinol Invest 2000;23:84-9.

28. Angulo M, Castro-Magana M, Mazur B, Canas JA, Vitollo PM, Sarrantonio M. Growth hormone secretion and effects of growth hormone therapy on growth velocity and weight gain in children with Prader-Willi syndrome. J Pediatr Endocrinol Metab 1996;3:393-9.

29. avies PSW, Evens S, Broomhead S, Clough H, Day JME, Laidlaw A, et al. Effect of growth hormone on height, weight, and body composition in Prader-Willi syndrome. Arch Dis Child 1998;78:474-6.
30. Carrel AL, Allen DB. Effects of growth hormone on body composition and bone metabolism. Endocrine 2000;12(2):16372

31. Eiholzer U, Blum WF, Molinari L. Body fat determined by skinfold measurements is elevated despite underweight in infants with Prader-Labhart-Willi syndrome. J Pediatr 1999;134:222-5.

32. Rosenbaum M, Gerner J, Leibel R. Effects of systemic (GH) administration on regional adipose tissue distribution in $\mathrm{GH}$ deficient children. J Clin Endocrinol Metab 1989;69:127481.

33. Cuneo RC, Judd S, Wallace JD, Perry-Keene D, Burger H, LimTio $\mathrm{S}$, et al. The Australian multicenter trial of growth hormone $(\mathrm{GH})$ treatment in $\mathrm{GH}$-deficient adults. J Clin Endocrinol Metab 1998;83:107-16.

34. Davies PSW, Joughin C. Assessment of body composition in the Prade-Willi syndrome. Am J Med Genet 1992;44:75-8.

35. Lee PD, Hwu K, Henson H, Brown BT, Bricker JT, Leblanc AD, et al. Body composition studies in Prader-Willi syndrome: effects of growth hormone therapy. Basic Life Sci 1993;60:201-5.

36. Fomon SJ, Hasche F, Ziegler EE, Nelson SF. Body composition of reference children from birth to age 10 years. Am J Clin Nutr 1982;35:1169-75.

37. Flier JS, Foster DW. Eating disorders. In: Wilson JD, Foster DW, Kronenberg HM, Larese PR. Williams textbook of endocrinology. $9^{\text {th }}$ ed. Philadelphia: Saunders, 1998. p. 1062.

38. Swaab DF. Prader-Willi syndrome and the hypothalamus. Acta Pediatr Suppl 1997;423:50-4.

39. Miller L, Angulo M, Price D, Taneja S. MR of the pituitary in patients with Prader-Willi syndrome: size determinations and imaging findings. Pediatr Radiol 1996;26:43-7.

40. Van Mil EG, Westerterp KR, Kester AD, Curfs LM, Gerver WJ, Schrander-Stumpel CT, et al. Activity related energy expenditure in children and adolescents wit Prader-Willi syndrome. Int Obes Relat Metab Disord 2000;24:429-34.

41. Haqq AM, Stadler DD, Jackson RH, Rosenfeld RG, Purnell JQ, Lafranchi SH. Effects of growth hormone on pulmonary function, sleep quality, behavior, cognition, growth velocity, body composition, and resting energy expenditure in Prader-Willi syndrome. J Clin Endocrinol Metab 2003;88(5):2206-12.

42. Rudling $M$, Norstedt G, Olivecrona $H$, Reihner E, Gustafsson JA, Angelin B. Importance of growth hormone for the induction of hepatic low density lipoprotein receptors. Proc Natl Acad Sci USA 1992;89(15):6983-7.

43. Lindgren AC, Ritzen EM. Five years of growth hormone treatment in children with Prader-Willi syndrome. Swedish Growth Hormone Advisory Group. Acta Paediatr Scand Suppl 1999;433:109-11.

44. Park MJ, Kim HS, Kang JH, Kim DH, Chung CY. Serum levels of insulin-like growth factor (IGF)-I, free IGF-I, IGF binding protein (IGFBP)-I, IGFBP-3 and insulin in obese children. $\mathbf{J}$ Pediatr Endocrinol Metab 1999;12:139-44.

45. National Cholesterol Education Program - Report of the expert panel on blood cholesterol levels in children and adolescents. Pediatrics 1992; 89:525.

\section{Endereço para correspondência:}

Jack Yung Kuo

Rua Pedroso Alvarenga 505, ap. 157

04531-011 São Paulo, SP

Fax: (1 1) 5052-0884

E-mail: jackyk@ig.com.br 